\title{
Towards Grounding Concepts for Transfer in Goal Learning from Demonstration
}

\author{
Crystal Chao, Maya Cakmak, and Andrea L. Thomaz \\ Robotics and Intelligent Machines, Georgia Institute of Technology, USA \\ cchao@gatech.edu, maya@cc.gatech.edu, athomaz@cc.gatech.edu
}

\begin{abstract}
We aim to build robots that frame the task learning problem as goal inference so that they are natural to teach and meet people's expectations for a learning partner. The focus of this work is the scenario of a social robot that learns task goals from human demonstrations without prior knowledge of high-level concepts. In the system that we present, these discrete concepts are grounded from low-level continuous sensor data through unsupervised learning, and task goals are subsequently learned on them using Bayesian inference. The grounded concepts are derived from the structure of the Learning from Demonstration (LfD) problem and exhibit degrees of prototypicality. These concepts can be used to transfer knowledge to future tasks, resulting in faster learning of those tasks. Using sensor data taken during demonstrations to our robot from five human teachers, we show the expressivity of using grounded concepts when learning new tasks from demonstration. We then show how the learning curve improves when transferring the knowledge of grounded concepts to future tasks.
\end{abstract}

\section{INTRODUCTION}

Our research focuses on the scenario of a social robot learning about everyday tasks demonstrated by a human. Although the robot may be capable of some skills a priori, such as pick-and-place manipulation, the exact task demands are ultimately determined by user preferences and the novel target environment. For example, cleaning a room or putting away groceries can vary significantly across different homes. In this situation, the robotics paradigm of Learning from Demonstration (LfD) allows naïve users to convey the desired task to the robot without any knowledge of programming [1], [2]. Users are able to teach the robot by demonstrating examples of the task.

What the robot learns about one task is generally only applied when performing that specific task. Transferring some knowledge to a future task could make the new task faster to teach. To achieve successful transfer, however, the robot must organize its sensory experiences from the demonstrations into grounded concepts - not arbitrary partitions of the sensory space, but ones that are semantically meaningful to the human. Such grounding has been explored computationally in [3] and with respect to word learning in [4]. In this paper, the approach we present does not rely on any linguistic structure but rather accomplishes such a grounding by leveraging the structure of the LfD process and the structure of human concepts. In our evaluation on data collected from naïve human subjects, we show that our system generalizes concepts that improve the efficiency of learning future tasks. Importantly, we also show that similar concepts are learned from different demonstrators.

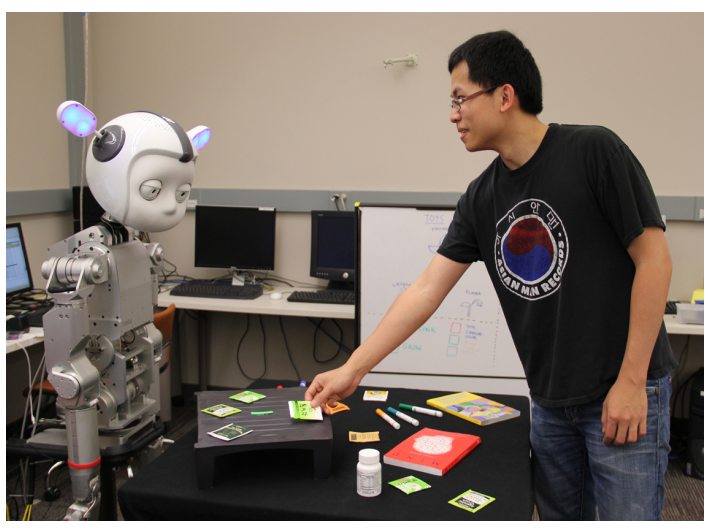

Fig. 1. Simon robot with human demonstrator at the tabletop workspace used in our experiments.

\section{A. The Structure of Learning from Demonstration}

How can the robot learner achieve the correct bias for grounding concepts when an infinite number of partitions of the sensory space are available? For one, observations about the world are not randomly distributed; for example, wings and the ability to fly frequently occur together (a property known as correlational structure [5]). But more significantly, the entire process of Learning from Demonstration offers a bias: the goal of the task, and the process of achieving it. In [6], Rosch discusses how levels of abstraction of objects fall out of their roles in achieving everyday goals. Goals and intentions form the basis of how humans interpret others' actions [7], [8], even in infants as young as nine months old [9], so it is sensible for a robot to interpret human demonstrations in terms of goal inference. Specifically in this interpretation, the robot learner should examine state changes and the resulting goal states as candidate grounded concepts.

We focus on goal learning rather than action execution in this paper. Our goal learning approach is compatible with LfD approaches that focus on trajectory learning [10], [11], action abstraction [12], [13], or action sequencing [14], [15]. Each of these levels of learning offers something unique to the robot, and they will be most powerful when used together.

\section{B. The Structure of Grounded Concepts}

One premise of our approach is that a robot's concept that is similar in structure to a human's concept will better match the human's expectations in interactions and task executions. 
One key characteristic of human concepts is that they exhibit graded structure [16], [17]. That is, human concepts have a central tendency, and people can identify prototypical examples as well as examples of higher or lower similarity. Typical category members are faster for category membership verification [18] and category member listing [19], and are also weighted more for induction [20].

There are several reasons that typicality could be important to a robot learner. The answer to whether the robot correctly executed the task could be better characterized as "almost" or "barely"; for example, a robot could place an object perilously close to the edge of a table. Another is that typicality could play a role in the sequence of examples that naïve human users naturally choose to teach to the robot. To capture the graded structure of human concepts, our approach supports a continuous representation at the lowest feature level and a Bayesian framework for learning the goal state.

Another important aspect of human concepts is their hierarchical nature. The basic level, describing entities such as "eggs" and "chairs," has been argued to be the most efficient level for clustering attribute information by maximizing within-category similarity against between-category similarity [6]. This stands in contrast with levels that are subordinate ("scrambled eggs" and "Windsor chairs") or superordinate ("food" and "furniture"). At a higher level, a special type of superordinate category called ad hoc categories describes groupings like "things to take on a camping trip," which can be imposed in the presence of goal contexts [21].

Any of these levels can be elicited during LfD and can be useful for future tasks. In grounding a concept at a certain level, the concept could later be construed as a discrete feature (with an underlying continuous representation) at the level of the task; for example, the presence or absence of a grounded "food" concept could be important to the task. By connecting concepts that share a sensory subspace in the order of increasing specificity or generality, a hierarchy of concepts can be constructed within our framework.

\section{APPROACH}

Although our work is motivated by human concept learning, our aim is not to create a computational model that attempts to explain all phenomena discovered by psychology, but rather to develop a robotics system with an awareness of these issues.

\section{A. System Overview}

Our learning system is implemented within the C6 software framework [22]. In this paper, we describe an extension to the C6 system in which concepts are grounded by constructing new percept functions online. The system learns from observed world state changes. Thus, the sensory-perception pipeline and state representation are the relevant aspects of the C6 system to this paper. In short, a world state in our system is a snapshot of a set of beliefs that exists at a certain time. A belief is a collection of activated percepts, each of which is a function of one or more sensory inputs.
Next we formalize these terms. At every time step $t$, the robot's sensory system represents a set $S(t)$ of data from its various sensory processes, where each $\mathbf{s}_{i} \in S$ is a vector of any length. The perception system at time $t$ describes a set of percepts $P(t)$, where each percept $p_{j} \in P$ represents a tuple structure $p_{j}(t)=\left\{f_{V_{j}}(S(t))=\mathbf{v}_{j}, f_{A_{j}}\left(\mathbf{v}_{j}\right)=a_{j}\right\}$. This tuple comprises an $m_{j}$-dimensional value $\mathbf{v}_{j}$ returned by evaluating a percept-specific value function $f_{V_{j}}$ on the sensory set, and a match probability $a_{j} \in[0,1]$ returned by evaluating an activation function $f_{A_{j}}$ on the percept value. Percept functions can vary significantly in structure, from simply returning the value from a sensor to being a complex function of multiple sensor values.

Percepts can also be continuous or discrete in value. Our system starts with an initial fixed set of continuous base percepts $P_{I} \subseteq P$. From $P_{I}$, a growing set of discrete grounded percepts $P_{I}^{\prime} \subseteq P$ is derived through the grounding process described later. For $p_{j} \in P_{I}$, the value $\mathbf{v}_{j}$ is continuous and the activation $a_{j}$ is equal to 1 . For $p_{j} \in P_{I}^{\prime}$, the value is 1 or 0 , and the activation $a_{j}$ is graded.

A belief $B(t)$ is a list of percepts that are activated at time $t$. The belief system maintains a belief set by integrating these percepts into discrete object representations using similarity metrics or tracking mechanisms. A world state $W(t)$ is the input to the task learner and refers to a snapshot of all beliefs at $t$. When analyzing state changes and modeling a task, activated percepts in these beliefs serve as features for the learner.

There has been debate about whether there is a difference between perceptual and conceptual categories. They have been described as contrasting mechanisms of object categorization in infants [23], but they could also form a single process that gets enriched over time [24]. Alternatively, conceptual categories might be learned via a bootstrapping mechanism starting with perceptual categories [25]. Because our concepts are created from percepts and operate at the perception system level, our system is akin to such a bootstrapping mechanism.

\section{B. Goal Learning from Demonstration}

Our system learns task goals from human demonstrations. Goals represent what must hold true about particular objects (beliefs) to consider the task achieved. In modeling the task goal, the robot needs to identify what percepts are relevant for inclusion as beliefs in the task, and what percepts need to change state to fulfill the goal. We define a set $E$ for potential expectations, which describe percept changes required to fulfill the task goal, and a set $C$ of potential criteria, the necessary prerequisites percepts for inclusion in the task. Thus, a learned task is executed by planning actions that fulfill percept expectations on objects that meet the percept criteria.

The start time $t_{S n}$ and end time $t_{E n}$ of the $n$th demonstration to the robot are demarcated using a graphical user interface or speech commands. For goal learning, the robot observes the world states at $t_{S n}$ and $t_{E n}$ to represent the $n$th demonstration $d_{n}=\left\{W\left(t_{S n}\right), W\left(t_{E_{n}}\right)\right\}$, and adds $d_{n}$ to the demonstration set $D$. In our LfD setup, objects that changed during the demonstration are considered noteworthy. In this 
paper, we consider demonstrations in which a single object is moved. First, beliefs and percepts are matched between $W\left(t_{S n}\right)$ and $W\left(t_{E n}\right)$. The belief that changed, $B_{\Delta}$, is found by identifying percepts that changed in value between $t_{E}$ and $t_{S}:\left|f_{V j}\left(S\left(t_{E n}\right)\right)-f_{V j}\left(S\left(t_{S n}\right)\right)\right|>\epsilon_{j}$, where the predetermined constant $\epsilon_{j}$ is a percept-dependent noise threshold. For each $p_{j} \in B_{\Delta}$ for which this holds true, $p_{j}\left(t_{E n}\right)$ is added to $E$. Then all percepts in $B_{\Delta}\left(t_{S_{n}}\right)$ are added to $C$.

For each continuous-valued $p_{j} \in E$, a model $M_{E_{j}}$ is constructed using an unsupervised learning method. We use principal components analysis (PCA) to reason about the data invariant to the directions selected for the original axes. So for all continuous-valued $m_{j}$-dimensional $p_{j} \in E$, the percept values $\mathbf{v}_{j_{1}} \ldots \mathbf{v}_{j_{n}}$ in the demonstration set $D$ are taken to form $m_{j} \times n$ matrix $\mathbf{M}_{j}$. The covariance matrix of the mean-subtracted demonstration data is then constructed: $\left.\mathbf{C}_{j}=\frac{1}{n}\left(\mathbf{M}_{j}-\overline{\mathbf{M}}_{j}\right) \cdot\left(\mathbf{M}_{j}-\overline{\mathbf{M}}_{j}\right)^{T}\right)$, on which the eigenvalue decomposition $\mathbf{V}_{j} \mathbf{C}_{j} \mathbf{V}_{j}^{T}=\mathbf{D}_{j}$ is performed to get eigenvectors (components) in the rows of $m_{j} \times m_{j}$ matrix $\mathbf{V}_{j}$ that correspond to eigenvalues in the $m_{j} \times m_{j}$ diagonal matrix $\mathbf{D}_{j}$. The same procedure is done for all continuous-valued $p_{j} \in C$.

This transformation of vector data maximizes the variability along the components, allowing one to reason about the data invariant to the directions selected for the original axes. For example, this approach can model variation in object locations along directions that don't align with the axes of the robot's world coordinate frame. The components in $\mathbf{V}_{j}$ corresponding to the highest eigenvalues in $\mathbf{D}_{j}$ indicate directions of higher freedom, and components that have small eigenvalues indicate tight constraints in the task. $\overline{\mathbf{M}}_{j}$ can be interpreted as the most representative or prototypical example of percept $p_{j}$.

The task goal is represented by two conjunctive hypotheses, $h_{C}$ and $h_{E}$. Before any grounding occurs, $h_{C}$ is the conjunction of all models $M_{C j}$ for all $p_{j} \in C$, and $h_{E}$ is the conjunction of all models $M_{E j}$ for all $p_{j} \in E$. When executing a task, beliefs are selected that satisfy $h_{C}$ and actions are selected to cause percept changes that satisfy $h_{E}$. Although the models are continuous, the decision to include a belief in the task is binary and is determined by thresholding on the goodness of fit. A value $\mathbf{v}$ being tested for fit is first projected into the model space, yielding new value $\mathbf{v}^{\prime}$. Assume the values along the diagonal in $\mathbf{D}_{j}$ are ordered, each being an eigenvalue matching an eigenvector $\mathbf{e}_{i}$ from the corresponding row $i$ in $\mathbf{V}_{j}$. The $i$ th entry of $\mathbf{v}^{\prime}$ is defined as:

$$
v_{i}^{\prime}=\left(f_{V j}(S(t))-\overline{\mathbf{M}}_{j}\right) \cdot \mathbf{e}_{i}
$$

The threshold for model membership $\tau_{i}$ at dimension $i$ is defined on the $i$ th standard deviation $\sigma_{i}$ of the reprojected demonstration data $\mathbf{V}_{j}^{T} \mathbf{M}_{j}$ and a constant $c$, which we have empirically set to 2.5 :

$$
\tau_{i}=c \cdot \sigma_{i}
$$

The binary fit in a criterion model $M_{C j}$ is determined by:

$$
M_{C j}\left(\mathbf{v}^{\prime}\right)= \begin{cases}1 & \text { if } \forall i, \mathbf{v}_{i}^{\prime}<\tau_{i}, \\ 0 & \text { otherwise. }\end{cases}
$$

Such binary values are used for the evaluation of $h_{C}$ and $h_{E}$ during task execution.

\section{Grounding Concepts}

The necessity of the binary decision on including a percept for task execution is the basis of grounding discrete concepts. These higher-level concepts are grounded from continuous percepts after a teacher finishes teaching a task. The representations are then reused for future task learning. For each model $M_{E j}$ and each model $M_{C j}$ of continuous-valued $p_{j} \in E, C$, a grounded percept $p^{\prime}$ is constructed from $p_{j}$ with a pointer back to $p_{j}$. Each grounded percept contains a new value function $f_{V}{ }^{\prime}$ and a new match function $f_{A}{ }^{\prime}$. The new value function simply uses Equation 3 to produce a binary value from the model. The new match function $f_{A}{ }^{\prime}\left(\mathbf{v}^{\prime}\right)$ produces a graded activation that represents the value's prototypicality:

$$
f_{A}^{\prime}\left(\mathbf{v}^{\prime}\right)= \begin{cases}\frac{1}{m_{j}} \sum_{i=1}^{m_{j}}\left(1-\frac{v_{i}^{\prime}}{\tau_{i}}\right) & \text { if } \forall i, v_{i}^{\prime}<\tau_{i}, \\ 0 & \text { otherwise. }\end{cases}
$$

Each new discrete grounded percept $p^{\prime}$ is added to the derived percept set $P_{I}^{\prime}$. When learning occurs in the new task, any $p^{\prime}$ with $a^{\prime}>0$ is included. The task goal is still represented by the conjunctive hypotheses $h_{C}$ and $h_{E}$. However, any grounded percept $p^{\prime}$ derived from a base percept $p_{j}$ is exclusive with and preferred over $p_{j}$ based on consistency with demonstrations. Consistency is defined on the Bayesian maximum likelihood probability of inclusion of the grounded percept as expressed by:

$$
\mathrm{P}\left(p^{\prime} \mid D_{1 \ldots n}\right)=\frac{\mathrm{P}\left(D_{1 \ldots n} \mid p^{\prime}\right) \mathrm{P}\left(p^{\prime}\right)}{\mathrm{P}\left(D_{1 \ldots n}\right)} \propto \alpha \cdot \mathrm{P}\left(D_{1 \ldots n} \mid p^{\prime}\right) \mathrm{P}\left(p^{\prime}\right)
$$

Equation 5 describes the probability that grounded percept $p^{\prime}$ is part of the task, given all of the demonstration data. $\mathrm{P}\left(D_{1 \ldots n} \mid p^{\prime}\right)$ is calculated as the fraction of demonstrations that are consistent with a hypothesis that includes $p^{\prime}$, based on the percepts activated during each demonstration. When $\mathrm{P}\left(D_{1 \ldots n} \mid p^{\prime}\right)=1$ for some $p^{\prime}$, the condition of maximal consistency, $p^{\prime}$ suppresses the $p_{j}$ from which it was derived because it can explain $D$. If there exist multiple $p^{\prime}$ derived from $p_{j}$ that are maximally consistent with the demonstrations, then this set of $p^{\prime}$ forms a version space $V$. The prior $\mathrm{P}\left(p^{\prime}\right)$ describes any biases about which percepts can be preferred; for LfD, we prefer more specific concepts, since they are the most conservative. If the demonstrations cause all grounded percepts to be removed from $V$ because none can account for $D$, then the fallback $p_{j}$ is used to capture the task structure. The grounding of $p_{j}$ from this task will then capture a different aspect of that sensory data than previous groundings. For example, a concept of "red" grounded from a base color histogram percept might not be useful in a task that requires 


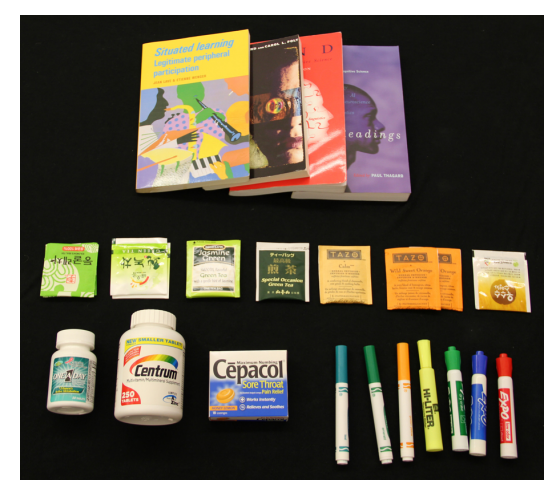

Fig. 2. Objects included books, tea bags, markers, and pill bottles.

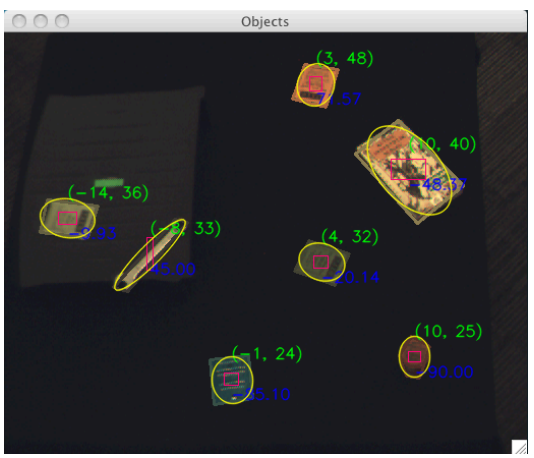

Fig. 3. Output of the vision system used for task demonstrations.

"orange," so the fallback percept of the color histogram would be used for the current task, and the concept of "orange" would then be grounded from the color histogram for future tasks.

\section{EXPERIMENT WITH HUMAN TEACHERS}

To evaluate our interactive learning approach, we tested our learner with five human teachers recruited from the campus community. Each subject was told to provide multiple demonstrations of five pick-and-place tasks to our upper-torso humanoid social robot "Simon" (Figure 1). In this particular experiment, the robot mostly functions as a passive observer except to structure the interaction with the human teacher using speech and eye gaze feedback. In other work, we focus more on the role that embodiment plays in interactive learning.

Each task demonstration involved moving a single object, and subjects provided eight demonstrations of each task. The tasks involved arranging various objects or classes of objects in the robot's tabletop workspace, analogous in structure to the scenario of teaching a robot how to tidy up a room. The tabletop workspace contained a transportable shelf as well as a variety of objects used in the tasks, including tea bags, pill bottles, books, and markers (see Figure 2). Prior to each task, the initial configuration of the workspace was randomized by an experimenter.

Subjects were asked to teach the following tasks:

- T1: Place markers on the shelf.

- T2: Place green tea bags on the right side of the table.

- T3: Place green tea bags on the shelf.
- T4: Place tea bags on the right side of the table.

- T5: Place objects from the shelf to the left side of the table.

Our system can be used with a variety of perceptual features, although the quality of the learned concepts naturally follows the quality of the features chosen. For this experiment, we used a number of simple features. Objects were detected through a fixed overhead camera, segmented using background subtraction, and localized in robot world coordinates using a fixed homography from the image plane to the table plane. The shelf in the workspace was detected and localized using a small bright-colored marker. The features computed for each segmented object were: (i) the height and the width of the bestfitting ellipse; (ii) the number of corners and the area of the simplified polygon contour of the segmented blob; and (iii) the hue histogram over the segmented blob. Each object used in learned tasks is represented by percepts on these low-level features as well as its location relative to the robot and relative to the shelf. Thus, $\left|P_{I}\right|=7$ for this experiment. Figure 3 shows sample output of the perception system.

The data collected was post-processed to select the object that changed most significantly in each example, although this procedure can be performed online. Due to noise and obstructions, some examples did not contain information. The resulting data sets included $25,35,37,44$, and 40 examples for T1-T5 respectively.

We used T1 and T2 as sources of continuous data for grounding discrete concepts. The concepts we hoped to obtain included representations for "green," "tea bags," "on the shelf," "right side of the table," and "left side of the table." We used the concepts grounded from $\mathrm{T} 1$ and $\mathrm{T} 2$ to evaluate if there was a change in learning speed for tasks T3-T5.

\section{RESUlTS}

In this section, we show that discrete concepts grounded from demonstrations of a set of tasks can be used as percepts in the hypotheses for future tasks. The grounded concepts match the expressivity of continuous models when the same concept is important to the task. Their discrete nature also reduces the minimum number of examples required for learning an adequate representation.

\section{A. Concepts Grounded}

Figure 4 shows visualizations for some of the concepts grounded from T1 and T2. Figure 4(a) shows concepts grounded from the location percepts. The gray border in each diagram shows the extent of the table or shelf, and the colored squares demarcate locations of objects in the demonstrations. For each pair of diagrams, the top row shows data from all demonstrators, and the bottom shows data from a single demonstrator. The gray region shows the learned concept model. Even the limited number of demonstrations given by an individual approximates the target concept almost as well as the combined data for the more basic-level concepts ("left", "right", "on shelf"), while having a poorer estimate for the superordinate concept of "anywhere on the table." 

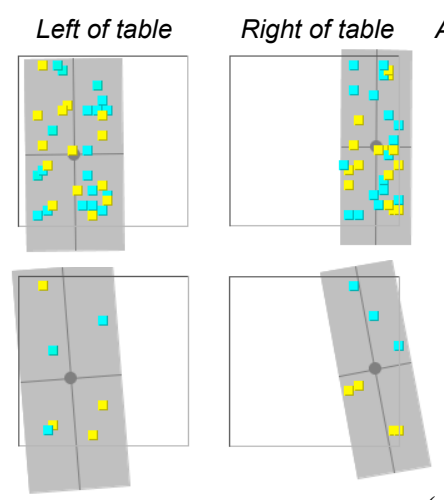

Anywhere on table

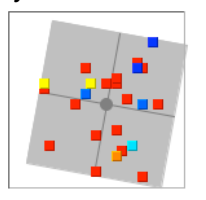

On the shelf
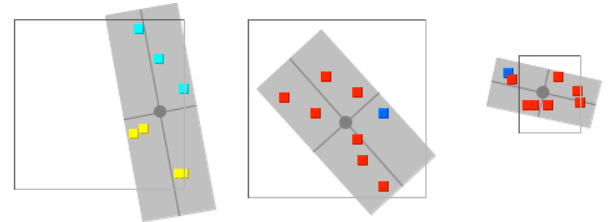

(a)

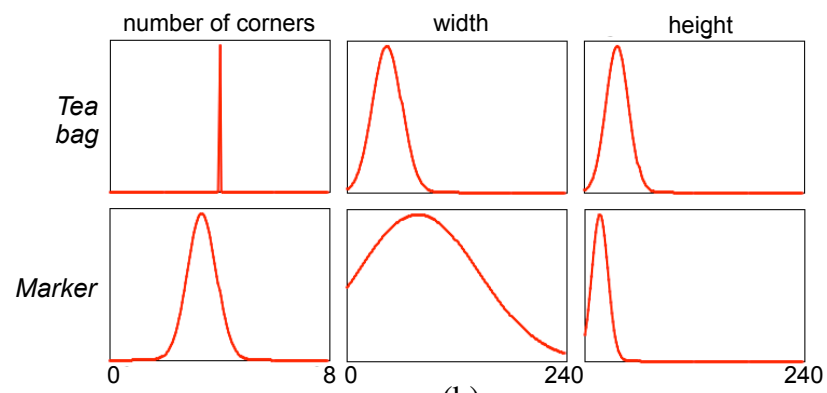

(b)

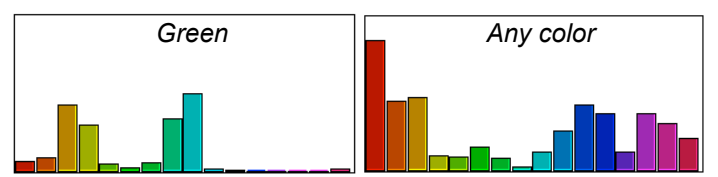

(c)

Fig. 4. Visualizations of demonstration data from T1 and T2. (a) Grounded location concepts. Colored squares show object locations from the demonstrations. For each pair of diagrams, the top row shows data from all demonstrators, and the bottom row shows data from a single demonstrator. The filled gray region demonstrates the concept learned. (b) Grounded concepts for number of corners, width, and height. Tea bags are squarish, and markers are long and thin, which is reflected in these models. (c) Grounded concepts for color. The color histogram for "any color" is much more varied than for the model of "green."

Figure 4(b) shows concepts derived from several other base percepts. The number of corners detected is tightly centered at 4 for the rectangular tea bags, but varies more for the cylindrical markers. The width and height models for tea bags are similar because of the relatively square proportions, but the markers have high variance since the width model is affected by orientation and perspective. The color histogram for "any color" is much more varied than for the model of "green" (Figure 4(c)).

The "left of table" concept was an interesting side effect that occurred in $\mathrm{T} 2$, in which green tea bags needed to go to the right side of the table. In order to show this, the human demonstrators selected green tea bags that were well on the left side of the table; none of the starting points were on the right side because those green tea bags were already fulfilling the necessary expectations. This bias from human teachers heightened the contrast for demonstrating the goal state, allowing the "left side of the table" concept to emerge
TABLE I

COVERAGE WITH AND WITHOUT GROUNDED CONCEPTS

\begin{tabular}{|c|c|c|}
\hline & Continuous Percepts & Grounded Concepts \\
\hline T1 & $88.00 \%$ & - \\
T2 & $85.71 \%$ & - \\
\hline T3 & $83.78 \%$ & $86.49 \%$ \\
T4 & $75.00 \%$ & $77.27 \%$ \\
T5 & $72.50 \%$ & $75.00 \%$ \\
\hline
\end{tabular}

as a criterion of $\mathrm{T} 2$ in addition to the "right side of the table" expectation that was the stated instruction to the subjects. This grounded $\mathrm{T} 2$ criterion could then be leveraged in learning $\mathrm{T} 5$.

\section{B. Goals Learned}

Next we characterize how well concepts grounded from T1 and $\mathrm{T} 2$ could be transferred to the new tasks T3-T5 that relied on the same underlying concepts. Note that although the tasks in this experiment were designed for transfer, the question that we pose here is whether or not demonstrations from naïve humans can generate grounded concepts that transfer to subsequent learning tasks.

Table I shows how well the goal learned actually fit the data with respect to matching both $h_{C}$ and $h_{E}$ in the goal representation. The "Continuous Percepts" column shows the percentage of data points that are accounted for by the continuous models using the threshold specified in Equation 2 as a cutoff, from only unsupervised learning. The "Grounded Concepts" column shows the percentage of data points that are accounted for using the highest-ranked grounded concepts in $V$ for all $p^{\prime}$ derived from $\mathrm{T} 1$ and $\mathrm{T} 2$.

For tasks T3-T5, the fit percentages are roughly comparable between learning using only continuous models and learning using grounded concepts. This indicates that the discrete representation using grounded concepts does not result in an overall loss of expressivity for the hypothesis space, as would usually occur from discretizing continuous spaces. This suggests that our approach produces a meaningful discretization.

Of course, if the coverage between two representations is comparable, there is no benefit to be seen from either over the other in terms of how well they capture task goals. The actual reason for performing the discretization of concepts from the continuous sensor data is for the speed of learning future tasks, which we discuss in the next section.

\section{Effect of Grounding New Features While Learning}

Figure 5 shows the effect of grounding on the learning curve for task T5. The $\mathrm{x}$-axis is the number of examples demonstrated, and the y-axis is coverage of all data for T5. The red dotted line indicates progress over number of samples using only continuous models, and the blue solid line indicates progress over number of samples using concepts grounded from $\mathrm{T} 1$ and $\mathrm{T} 2$.

The chart in the upper left shows the curve over all teachers' demonstrations, and the other charts show progress for individual teachers. Progress is achieved more quickly using grounding, making it a useful way to gain efficiency in 

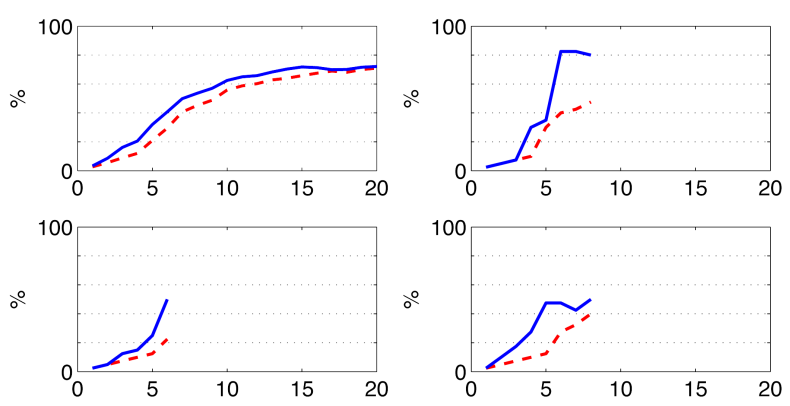

Fig. 5. Learning curve for combined data (top left) and three sample individual teachers on the transfer task T5. The $\mathrm{x}$-axis is the demonstration number $n$, and the $\mathrm{y}$-axis is the coverage. The coverage of the combined data is the average across 20 random orderings. The dashed line shows learning without grounded concepts, and the solid line shows learning using grounded concepts. In the combined data, the two approaches converged after approximately 17 examples (out of a total of 40 ).

teaching and learning. This is especially visible in the smaller sample sizes for individual teachers. This result makes sense when the discretization matches ground truth. For example, the shelf is a rectangular object, so the concept of "on the shelf" requires a minimum of four data points in order to form an accurate model. However, matching a previously grounded "on the shelf" concept only requires a single data point that fits inside the learned model.

The grounded goal for T5 included concepts like "on the shelf" and "left side of the table." However, not all of the grounded features are necessarily interesting or important. In fact, some of them are so varied that they can basically be described as "anywhere on the table" or "any color," which are concepts that get matched too easily. Because this presents a scaling issue, an important direction for future work is pruning concepts that don't have valuable meaning. One method is to consider the relationship between the variance of the data and the limits of the feature values. Another way could be through active learning, as we have previously explored in [26].

\section{CONCLUSION}

We present a robot goal learning system that can ground discrete concepts from continuous perceptual data using unsupervised learning. The higher-level grounded concepts enable our robot Simon to represent and reason about new tasks more quickly. Using data collected from five human demonstrators, we show that this system and representation are appropriate when learning from demonstrations provided by human teachers who are non-experts in robotics. We also show that the ability to ground concepts results in increased efficiency when transferring this knowledge to learning future tasks.

\section{REFERENCES}

[1] A. Billard, S. Calinon, R. Dillmann, and S. Schaal, "Robot programming by demonstration," in Handbook of Robotics, B. Siciliano and O. Khatib, Eds. Springer, 2008, ch. 59, pp. 1371-1394.

[2] B. D. Argall, S. Chernova, M. Veloso, and B. Browning, "A survey of robot learning from demonstration," Robotics and Autonomous Systems, vol. 57, no. 5, pp. 469-483, 2009.
[3] J. Stober and B. Kuipers, "From pixels to policies: a bootstrapping agent," in IEEE International Conference on Development and Learning (ICDL), 2008, pp. 103-108.

[4] D. K. Roy, "Learning words from sights and sounds: A computational model," Ph.D. dissertation, Massachusetts Institute of Technology, 1999.

[5] E. H. Rosch, C. B. Mervis, W. D. Gray, D. M. Johnson, and P. BoyesBraem, "Basic objects in natural categories," Cognitive Psychology, vol. 8, pp. 382-439, 1976.

[6] E. H. Rosch, "Principles of categorization," in Cognition and Categorization, E. H. Rosch and B. B. Lloyd, Eds. Hillsdale, NJ: Lawrence Erlbaum Associates, 1978, pp. 27-48.

[7] A. L. Woodward, J. A. Sommerville, and J. J. Guajardo, "How infants make sense of intentional actions," in Intentions and Intentionality: Foundations of Social Cognition, B. Malle, L. Moses, and D. Baldwin, Eds. Cambridge, MA: MIT Press, 2001, ch. 7, pp. 149-169.

[8] D. A. Baldwin and J. A. Baird, "Discerning intentions in dynamic human action," Trends in Cognitive Sciences, vol. 5, no. 4, pp. 171-178, 2001.

[9] G. Csibra, "Teleological and referential understanding of action in infancy," Philosophical Transactions of the Royal Society B: Biological Sciences, vol. 358, no. 1431, pp. 446-458, March 2003.

[10] S. Calinon and A. Billard, "A probabilistic programming by demonstration framework handling skill constraints in joint space and task space," in IEEE/RSJ International Conference on Intelligent Robots and Systems (IROS), 2008, pp. 367-372.

[11] P. Pastor, H. Hoffmann, T. Asfour, and S. Schaal, "Learning and generalization of motor skills by learning from demonstration," in IEEE International Conference on Robotics and Automation (ICRA), 2009, pp. 1293-1298.

[12] O. C. Jenkins and M. J. Matarić, "Performance-derived behavior vocabularies: Data-driven acquisition of skills from motion," International Journal of Humanoid Robotics, vol. 1, no. 2, pp. 237-288, 2004.

[13] D. Kulic, W. Takano, and Y. Nakamura, "Incremental learning, clustering and hierarchy formation of whole body motion patterns using adaptive hidden markov chains," International Journal of Robotics Research, vol. 27, no. 7, pp. 761-784, 2008.

[14] R. Zöllner, M. Pardowitz, S. Knoop, and R. Dillmann, "Towards cognitive robots: Building hierarchical task representations of manipulations from human demonstration," in IEEE International Conference on Robotics and Automation (ICRA), 2005, pp. 1535-1540.

[15] N. Nicolescu and M. J. Matarić, "Natural methods for robot task learning: Instructive demonstration, generalization and practice," in Second International Joint Conference on Autonomous Agents and Multi-Agent Systems (AAMAS), 2003, pp. 241-248.

[16] L. J. Rips, E. J. Schoben, and E. E. Smith, "Semantic distance and the verification of semantic relations," Journal of Verbal Learning and Verbal Behavior, vol. 12, pp. 1-20, 1973.

[17] E. H. Rosch, "On the internal structure of perceptual and semantic categories," in Cognitive development and the acquisition of language, T. E. Moore, Ed. Academic Press, 1973, pp. 111-144.

[18] M. McCloskey and S. Glucksberg, "Decision processes in verifying category membership statements: Implications for models of semantic memory," Cognitive Psychology, vol. 11, pp. 1-37, 1979.

[19] C. B. Mervis, J. Catlin, and E. Rosch, "Relationships among goodnessof-example, category norms, and word frequency," Bulletin of the Psychonomic Society, vol. 7, pp. 283-284, 1976.

[20] D. N. Osherson, E. E. Smith, O. Wilkie, A. López, and E. Shafir, "Category-based induction," Psychological Review, vol. 97, pp. 185200, 1990.

[21] L. W. Barsalou, "Ad hoc categories," Memory \& Cognition, vol. 11, no. 3, pp. 211-227, 1983.

[22] B. Blumberg, M. Downie, Y. Ivanov, M. Berlin, M. Johnson, and B. Tomlinson, "Integrated learning for interactive synthetic characters," in Annual Conference on Computer Graphics and Interactive Techniques (SIGGRAPH), 2002, pp. 417-426.

[23] J. M. Mandler, "Perceptual and conceptual processes in infancy," Journal of Cognition and Development, vol. 1, no. 1, pp. 3-36, 2000.

[24] P. C. Quinn and P. Eimas, "The emergence of category representations during infancy: Are separate perceptual and conceptual processes required?" Journal of Cognition and Development, vol. 1, no. 1, pp. 55-61, 2000.

[25] G. L. Murphy, The Big Book of Concepts. MIT Press, 2002.

[26] M. Cakmak, C. Chao, and A. L. Thomaz, "Designing interactions for robot active learners," IEEE Transactions on Autonomous Mental Development, vol. 2, no. 2, pp. 108-118, June 2010. 